\title{
Adopción de innovaciones en la agricultura familiar latinoamericana
}

\author{
Adoption of innovations in Latin American family farming
}

\author{
Jorge Luis Triana Riveros ${ }^{1}$ \\ Karim Marini Thomé ${ }^{2}$
}

Fecha de recepción: 24-03-2020

Fecha de aceptación: 16-3-21

\begin{abstract}
Resumen
En este artículo se realizó un estudio del proceso para adoptar una innovación en la agricultura familiar, identificando variables del entorno organizacional e institucional que influencian en el proceso de adopción. El artículo se desarrolló mediante una revisión de literatura, analizando las principales variables que afectan la adopción, con el fin de contribuir al fortalecimiento de los procesos de innovación en la agricultura familiar; y así, aprovechar las innovaciones creadas por nuestros centros de investigaciones, universidades y sector privado-público. Se concluyen que es de gran importancia que las entidades de investigación realicen alianzas con instituciones intermediarias para difundir los conocimientos adquiridos con el objetivo de no tener fracaso en las adopciones.
\end{abstract}

Palabras claves: innovación, adopción, agricultura familiar, agricultura, Latinoamérica.

\begin{abstract}
In this article, a study of the process to adopt an innovation in family farming was carried out, identifying variables of the organizational and institutional environment that influence the adoption process. The article was developed through a literature review, analyzing the main variables that affect adoption, in order to contribute to the strengthening of innovation processes in family farming; and thus, take advantage of the innovations created by our research centers, universities and the private-public sector. They conclude that it is of great importance that research entities make alliances with intermediary institutions to disseminate the knowledge acquired with the aim of not having failure in adoptions
\end{abstract}

Keywords: innovation, adoption, family agriculture, agriculture, Latin America.

\footnotetext{
1 Ingeniero Agrónomo de la Universidad de los Llanos, Magister en Agronegocios en la Universidad de Brasilia y estudiante de doctorado en Política Social en la Universidad de Brasilia, Brasil. Correo electrónico: jorge.triana@unillanos.edu.co

2 Administrador del Centro Universitario UnirG, Ingeniero Agrónomo de la Universidad Federal do Tocantins, Magister en Agronegocios de la Universidad de Brasilia, Brasil Doctor en Administración de la Universidad de Lavras y realizó Pos doctorado en Sotckholm School of Economics y Uppsala University. Correo electrónico: thome@unb.br.
} 


\section{Introducción}

En los países en desarrollo la agricultura es un factor importante para el crecimiento económico, por lo tanto, para que una industria, empresa o cooperativa presente mayor eficiencia y sea ampliamente reconocida, es necesario aplicar innovaciones en el proceso de producción para favorecer la productividad. Un ejemplo propio de adopción de innovación en la agricultura convencional fue implementar la Revolución Verde en la década de 1960, que en resumen se trataba de un manejo fitosanitario riguroso con la aplicación de productos químicos en los cultivos, práctica que todavía es bastante utilizada (Gonçalves et at., 2021).

Para agregar a la idea anterior, en un texto de la Empresa Brasileña de Investigación Agropecuaria EMBRAPA (2003) se encuentra la siguiente afirmación:

[...] En los sistemas agroindustriales de gran tamaño, las estructuras de coordinación se establecen y por sí solo adquieren capacidad para determinar la dinámica de la innovación y la gestión tecnológica mientras que, en la agricultura familiar, hay necesidad de encontrar formas, mecanismos organizativos e institucionales capaces de proporcionar esta gestión tecnológica, siendo este uno de los principales desafíos del proceso. (EMBRAPA, 2003, p.20).

Por este motivo, se considera que la agricultura familiar es un grupo de productores de mediano o pequeño porte, siendo actores sociales de la agricultura moderna compuesta por una unidad familiar (padre, madre e hijos), donde el trabajo en conjunto tiene la capacidad de adaptarse a las innovaciones y exigencias de la modernización en los mercados (Wanderley, 2013).

Por otro lado, Abramovay (1998) afirma que la agricultura familiar expone un sector social apto para equilibrar los ambientes organizacionales e institucionales simbolizando la probabilidad de construir mejores condiciones de vida. Una cuestión que surge es la siguiente pregunta: ¿Por qué el gran productor tiene mejor adopción de innovaciones? Balsan (2006) afirma que el procedimiento de modernización por medio de la adopción de innovaciones, en cierto grado desligó a las clases menos favorecidas, el fracaso de los productores familiares con poco poder adquisitivo y con área restringida, por el hecho de no tener recursos financieros suficientes para adoptar innovaciones.

En este sentido, la innovación es sinónima de cambio, mudanzas, adaptación a una nueva etapa para perdurar en los mercados, por tal motivo, si no se renueva la empresa, persona o cooperativa; va a ser alcanzada por sus competidores, saliendo fácilmente del mercado. Según Gopalakrishnan y Damanpour (1997) la innovación promueve el procedimiento de ajuste de cambios ilimitados y cumple un papel importante en incentivar la economía a través de la mejora del rendimiento de las empresas en la construcción de la competitividad industrial. Esta presenta las siguientes dimensiones: fases de la innovación, modelos de difusión, innovación en el ambiente organizacional, características de las innovaciones, características de los innovadores y contexto ambiental institucional.

Por tal motivo, Hall et al. (2001) afirman que para asumir las innovaciones en la agricultura es necesario conocer a fondo los problemas de los productores, realizando investigaciones agrícolas, analizando la relación insumo/producto y desarrollar un buen plan de difusión. Pues, al adoptar una innovación, el productor aporta efectos e impactos en el ámbito social y económico, lo que suscita entender las etapas que pasa desde la innovación a la adopción.

Según North (1991) el ambiente organizacional e institucional influye en adoptar innovaciones en la agricultura familiar, donde el ambiente institucional abarca normas, impuestos, créditos, asistencia técnica e investigaciones, percibiendo así características para lograr dicha innovación. En este sentido, el ambiente organizacional está formado por instituciones públicas o privadas que apoyan al proceso en cuestión, implantando normas en un ambiente institucional (Lima et al., 2001). Por lo tanto, el objetivo de este 
artículo es analizar las variables de ambiente organizacional e institucional que inciden en la adopción de innovaciones en la agricultura familiar latinoamericana.

\section{Metodología}

$\mathrm{Al}$ tener en cuenta que las adopciones de innovaciones son bajas en los productores familiares, se realizó una revisión de literatura sobre autores que han estudiado la innovación, con el fin de cumplir el objetivo propuesto, se establecieron los descriptores innovación, adopción, agricultura familiar, Latinoamérica. La investigación fue realizada en el Portal de Periódicos de la CAPES, Scientific Eletronic Library (SCIELO), science direct y google académico, analizando los aportes teóricos contenidos en los documentos con mayor a 10 citaciones en Google académico y con fechas recientes de publicación.

\section{Resultados y discusión}

En primer lugar, se investigó el concepto de agricultura familiar, donde Chayanov (1975) estableció el concepto de unidad campesina a principios del siglo XX como integración de la producción agrícola que puede ser eficiente y competitiva, basada en una racionalidad distinta, en la que el objetivo fundamental es apuntar a las necesidades de consumo, más que el deseo de obtener beneficios. En este sentido, Ploeg (2009) destaca el desempeño de la actual agricultura campesina, presentando características familiares, en el momento de confrontar las presentes condiciones de colapso económico y financiero global.

El modelo de producción campesina necesita ser valorado como uno de los principales elementos para perpetrar las adversidades actuales, lo que establece la existencia de diseños de agriculturas dualistas que pueden ser identificados en tres categorías: agricultores familiares, grandes productores, y campesinos; siendo los dos últimos diferenciados por tener mayor adopción de innovación industrial (Ploeg, 2009).
La desigualdad del proceso de modernidad se puede explicar a partir del desarrollo de adopción y ampliación de las innovaciones. En pocas palabras, el productor tendrá que armar objetivos que se anteponen al método a ser adoptado en el proceso (Cuadro 1) (Gerardi, 1980 apud Balsa, 2006). Por otro lado, Valentino (2007) discute que los productores familiares pueden adaptarse a las innovaciones, y al crear cooperativas traen beneficios a algunas particularidades, como ahorrar costos de transacciones, ayudar a crear poder compensatorio y recurrir a una gama diversificada de estrategias organizacionales. De este modo, se logra entonces desempeñar un papel importante en las adopciones y, por lo tanto, se generan incentivos sistemáticos para los productores al ser parte de dichas cooperativas.

\section{Cuadro 1 Principales desafíos que esclarecen a diferenciación regional de la modernización (adopción de innovaciones.}

\begin{tabular}{|l|l|}
\hline Desafíos & Principales diferenciaciones \\
\hline Psicológicos & $\begin{array}{l}\text { Son consideraciones que el agricultor toma } \\
\text { como riesgos e incertidumbres para adoptar } \\
\text { una práctica nueva. }\end{array}$ \\
\hline Económicos & $\begin{array}{l}\text { Asumir nuevas técnicas sujetas al capital que } \\
\text { el agricultor dispone para invertir. }\end{array}$ \\
\hline $\begin{array}{l}\text { De información o } \\
\text { culturales }\end{array}$ & $\begin{array}{l}\text { La poca o total ausencia de información, lo } \\
\text { que limita o imposibilita la ampliación de la } \\
\text { modernización. }\end{array}$ \\
\hline
\end{tabular}

Fuente: Adaptado (Gerardi, 1980 apud Balsa, 2006).

Del mismo modo, se realizó el análisis de la literatura consultada, adaptando un modelo del proceso de la adopción de una innovación en la agricultura familiar (Figura 1), de esta manera explicando cada sección en este capítulo. 
Figura 1. Proceso de la innovación a la adopción en la agricultura familiar.

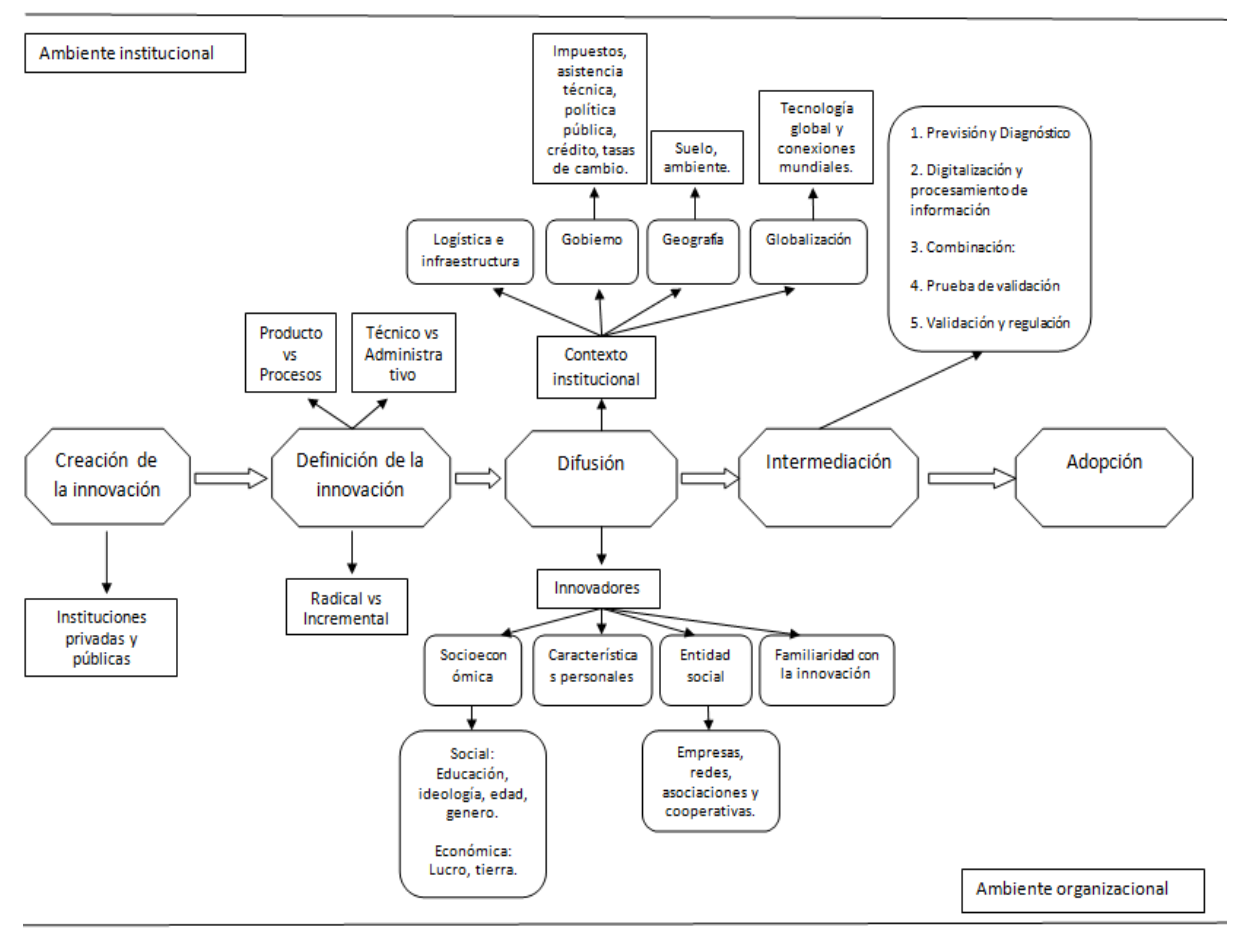

Fuente: (Diversos autores: adaptación de la literatura consultada).

\subsection{Fases de la innovación}

Gopalakrishnan y Damanpour (1997) apuntan que es importante recordar de dónde se genera la innovación y tener claro las etapas, donde se admite que cada fase tiene como función suprimir ambigüedades sobre la innovación. Después de identificar dicha innovación, se continua con el proceso de la adopción, que es un procedimiento organizacional que puede ser un obstáculo directamente a los sistemas técnicos y sociales de una organización. Estos dos elementos se evalúan por el grado de integración en las organizaciones, se presentan así las siguientes etapas.

3.1.1 Análisis de nivel: es un análisis que tiene en cuenta cuatro conceptos: (a) Industria (identificar las diferencias en los patrones del desarrollo y en la magnitud de la innovación en la interacción de las industrias), (b) Organización (características textuales, organizacionales, estructurales y de comportamiento que divide organizaciones innovadoras y no innovadoras); (c)
Sub unidades organizacionales (analiza los fenómenos asociados con la innovación y enfatiza cuestiones de metodología); (d) Innovación (apuntan al análisis de los costos, ventajas, complejidad que influyen en las tasas de difusión de una innovación). En este sentido se puede identificar que las innovaciones presentan tres tipos, tal y como se muestra en la figura 2.

\section{Figura 2. Tipos de innovaciones.}

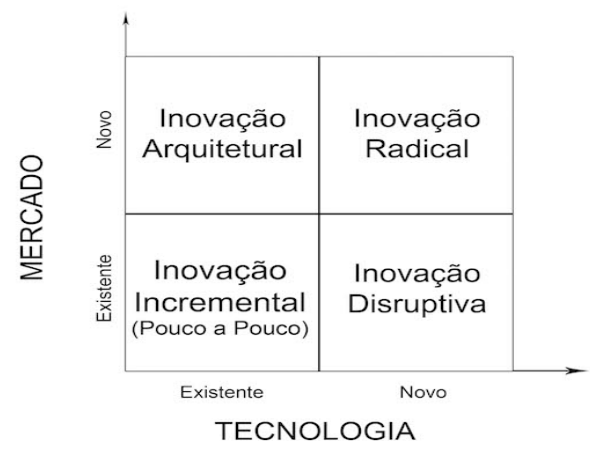

Fuente: Mahala, (2021). 
En este sentido se consiguió identificar que la innovación producto vs proceso, es una relación entre las áreas y las actividades que pueden afectar la innovación. También se identificó la radical vs incremental, donde la innovación radical presenta cambios fundamentales en una organización, es decir, hace desvíos de las prácticas actuales. Por su parte, la incremental hace desvíos marginales de las prácticas existentes, como ejemplo de innovación incremental se puede citar el caso de la producción de leche donde los cambios son lentos o graduales en función al mercado, de acuerdo con Gardner y Oliveira (1984): "La principal inquietud de los investigadores, asistentes técnicos en la agricultura es la lenta o no adopción de una innovación creada" (p.246). Además, se identificó lo técnico vs administrativo, donde se ven relacionados con todo el núcleo técnico y se colabora para crear una distinción en la estructura tecnológica y social.

\section{Modelos de difusión de la innovación}

Cuando se demarca el término de difusión se trata de simplemente difundir, propagar y ampliar una idea. Para Rogers (1995): "la difusión de una innovación es la metodología que se transfiere a través de canales de comunicación en un determinado intervalo de tiempo entre los miembros de un sistema social” (p.5), y según Wejnert (2002) la influencia y la comunicación, pueden desviar la toma de decisiones de un agente al adoptar una innovación, agrupando las variables difusoras en el siguiente componente: Innovación en el contexto organizacional, se trata de centrarse en la competitividad y eficiencia de una empresa con el fin de mantener el alto rendimiento y competir en su medio, estando vinculada a los eventos ambientales como mecanismos de respuesta.

Asimismo, la gestión para innovar presenta dos factores: velocidad de adaptamiento (reflejo de la rapidez de una organización al adoptar una renovación que puede influir en el éxito en esa innovación) y amplitud de la adopción (la organización tiene que institucionalizar los procesos para mantener la flexibilidad y de esta forma propagar la innovación) (Gopalakrishnan, Damanpour, 1997).
En el caso de las empresas de investigación en el área de la agricultura EMBRAPA, Corporación Colombiana de Investigación Agropecuaria, Instituto Nacional de Tecnología Agropecuaria (INTA), Universidades, entre otras, se tienen un papel importante en adoptar actualizaciones, porque desarrollarán mejores técnicas de producción, creando competitividad. En este sentido la asistencia técnica es una importante herramienta en el proceso de adopción para cualquier productor, porque esta tendrá en mano el mejor método para que el agricultor adopte la innovación por medio de técnicas más adecuadas a sus necesidades.

Es importante recordar que las cadenas productivas son la base de estudio de los diferentes productos agropecuarios donde Thomé et al., (2020) afirman que estas cadenas cada día están siendo más innovadoras y debido a esta necesidad de agregar valor y la convergencia de intereses, la convivencia coordinativa es la más prevalente en la literatura, aunque no se reconoce explícitamente. Por estos motivos, esta interrelación de las cadenas conduce a la plasticidad de la cadena de suministro, lo que enfatiza la capacidad de realizar cambios rápidamente en dichos suministros, con el fin de adaptarse a cambios significativos, creando así, la sostenibilidad como un cambio relevante en el entorno empresarial siendo el motor de coexistencia.

Por esta razón, se está de acuerdo con Rodrigues et al., (2021) donde afirman que la interdependencia de los stakeholder es causada por las prácticas, posibilitando establecer un entorno de colaboración al permitir gestar nuevas destrezas, ya que por ejemplo, las praxis de intercambio de animales generan prácticas de intercambio de alimentos y las praxis de intercambio de trabajo generan habilidades y conocimientos, mejorando la gestión de recursos y la gobernanza que inicialmente son requeridas para adoptar una innovación.

En cuanto a las características de las innovaciones estas han sido poco estudiadas, pero son determinantes para que ocurran las circunstancias para adoptar la innovación. Wejnert (2002) se enfatiza en las características de las innovaciones en cuanto a sus influencias públicas y privadas. Siendo las públicas adopciones que conducen 
a los avances históricos en una comunidad (leyes, políticas de confort, regulación internacional) que tiene por objetivo buscar el desarrollo agrícola, por ejemplo, el Programa Nacional de Fortalecimiento de la Agricultura Familiar (PRONAF) que se fija financiar proyectos de innovaciones para promover la generación de ingresos a los productores.

En referencia a las influencias privadas, son aquellas que pueden afectar el bienestar de los productores y emprendimientos de menor porte, considerando que las grandes empresas adoptan innovación de alto costo, citando como ejemplo las soluciones mecánicas y tecnológicas desarrolladas para granjas lecheras, que son de coste elevado, y por ende, difíciles de ser adoptados por productores familiares individuales (Organización de las naciones unidas para alimentación y agricultura, 2013), lo que exigirá, en el caso de adoptar la misma innovación, que los productores de menor porte se organicen por medio de cooperativas, asociaciones o condominios.

Es importante resaltar que en las características de las innovaciones es indispensable tener claro los conceptos costos/beneficios. De acuerdo con Wejnert (2002), los costos pueden relacionarse con pérdidas o ganancias, sean monetarios (financieros) o no monetarios (tiempo, estructura familiar, social, reputación) y están asociados a una adopción, por lo que los costos a menudo tienden a inhibir la adopción de las innovaciones.

Algunos elementos a tomar en cuenta también son:

3.2.1 Innovación en el contexto institucional: Es importante analizar cómo se desarrolla la difusión de las innovaciones. Wejnert (2002) muestra cuatro subgrupos de variables que son intrínsecas en el contexto ambiental, son: geográfica, cultura social y condiciones políticas, relatadas a continuación.

3.2.2 Geografía: Esta variable tiene una fuerte influencia en la aplicabilidad de la adopción, según el autor anteriormente mencionado las estructuras como suelo, clima, ambiente pueden ejercer cambio en la opción de adopción de una innovación. Por ejemplo, en la investigación de Orduz-Rodríguez et al. (2006) sobre el comportamiento de la mandarina arrayana en seis patrones en suelos con alta acidez en los Llanos Orientales Colombianos, resulta que el patrón Citrumelo Swingle presentó variables de producción (precocidad, peso, calidad de fruto) mejores y más altos, y por ese motivo se trasladó a los productores familiares de aquella región. Sin embargo, dicha innovación no cabría ser adoptada en regiones con características ambientales distintas de los Llanos Colombianos porque son patrones no adaptados a otras zonas.

3.2.3 Cultura social: En este subgrupo se estudia las influencias en la aplicación de la innovación y algunas variables como valores, normas, lenguaje, religión, cultura, socialización y género, que pueden influir en la adopción de las innovaciones. Por ejemplo, en el estudio de Moraes (2020) de producción de carne bovina (vacas) afirmaron que la carne tiene un potencial a ser explorado, siendo necesario las adopciones de estrategias competitivas, como marcas de calidad, llevando la diferenciación de los productores para atender los nichos de mercados donde estos rendimientos se pueden ver en los mejores promedios de materia seca. Además, en el segundo año la ganancia de peso de las vacas fue mayor con esa especie, sin embargo, en la India este procedimiento no tendría éxito entre los productores familiares, debido a las cuestiones culturales y religiosas. La religión predominante es el hinduismo y por la creencia, los fieles no consumen carne de vaca. En esta situación se percibe cómo una cultura influye en la adopción de una innovación.

Ahora bien, las relaciones sociales se han vuelto más complejas, pero es exactamente por la aparente separación entre la economía y los bienes extraeconómicos, impidiendo la percepción de una manera a instancias del capital, donde se puede afirmar que aunque los problemas tales como género y etnia, son importantes, y que cualquier investigador tiene que prestar atención a estos puntos, tal y como se están debatiendo, acaban moldeados antes los nuevos modelos innovadores (Cavalcanti, 2020). 
En este sentido, dirigiéndonos al género, las mujeres son uno de los objetivos de las actividades reproductivas, reforzando las actividades al hogar y a la familia, separándolos de los espacios público y político y la posibilidad de percibir y ser percibido como un individuo, social y política (Moraes, 2021). Aunque el ambiente organizacional delimita estos elementos como los roles de género dentro de la familia y la comunidad, las mujeres siguen sin tener estas importancias en las sociedades, existiendo de este modo, una limitante para la adopción de las innovaciones.

En estas perceptivas, los valores son importante en el momento de una adopción. Para que estos permanezcan inalterados y puedan transmitirse de generación en generación, es necesaria la parte educativa y el cuidado de estos principios, puesto que son fundamentales para lograr la continuidad de los jóvenes rurales en el campo, para un adecuado desarrollo (Triana, 2019).

Por estas razones, el género y los valores tienen una importancia alta en la influencia de la difusión de las adopciones para innovar, según Van Everdingen y Waarts (2003), los valores culturales varoniles se inclinan hacia la eficiencia y la competencia, es decir, el éxito ayuda a producir ilusiones con más valor de heroísmo, y la mujer fomenta el cuidado, la intuición, la precavida y la solidaridad, por lo que la productora rural adopta innovación un poco más despacio que el hombre, en virtud de que el reconocimiento no es su máxima prioridad. Esta data se corrobora en la investigación de Lima et al., (2013), al aseverar cómo se percibe la importancia en el campo brasileño en referencia al género una vez que este presenta estar fuertemente asociado a la conquista de posiciones de poder, con fuerte tendencia a la sucesión masculina.

\subsection{Características de los innovadores}

En general, los innovadores serán siempre actores exitosos, pero se percibe que algunos productores familiares son más receptivos que otros y por ese motivo son los primeros en adoptar novedades, mientras que otros productores esperan hasta minimizar las incertidumbres asociadas a las nuevas adopciones. Según Wejnert (2002) las características de los actores pueden intervenir en la asimilación de los beneficios para adaptarse, por lo que delimita las características más fundamentales de estos sujetos. Obsérvese en el cuadro 2.

\section{Cuadro 2 . Características de los innovadores.}

\begin{tabular}{|l|l|}
\hline $\begin{array}{l}\text { Características de } \\
\text { los innovadores }\end{array}$ & Concepto \\
\hline $\begin{array}{l}\text { Características del } \\
\text { Estado }\end{array}$ & $\begin{array}{l}\text { Posición de un actor dentro de una } \\
\text { población (gobierno). }\end{array}$ \\
\hline $\begin{array}{l}\text { Entidad social de } \\
\text { los innovadores: }\end{array}$ & $\begin{array}{l}\text { Resultados de adopción macro (patentes, } \\
\text { modelos agrarios) y micro (redes de } \\
\text { firma, organización, cooperativas). }\end{array}$ \\
\hline $\begin{array}{l}\text { Familiaridad con la } \\
\text { innovación }\end{array}$ & $\begin{array}{l}\text { Depende de la institucionalización, } \\
\text { observación y puede ser radical o } \\
\text { gradual. }\end{array}$ \\
\hline $\begin{array}{l}\text { Características } \\
\text { socioeconómicas }\end{array}$ & $\begin{array}{l}\text { Educación, ideologías, tamaño de la } \\
\text { propiedad, edad, renta, entre otras, } \\
\text { pueden causar alta o baja adopción de } \\
\text { innovación. }\end{array}$ \\
\hline $\begin{array}{l}\text { Características } \\
\text { personales }\end{array}$ & $\begin{array}{l}\text { Confianza para tener una buena tasa de } \\
\text { adopción. }\end{array}$ \\
\hline $\begin{array}{l}\text { Posición de redes } \\
\text { sociales }\end{array}$ & $\begin{array}{l}\text { Las redes segmentadas presionan a sus } \\
\text { miembros para uniformizar las prácticas. }\end{array}$ \\
\hline
\end{tabular}

Fuente: adaptado de Wejnert (2002)

Según Souza Filho et al. (2011) el tamaño de la propiedad en referencia a la adopción de innovaciones depende de aspectos técnicos, económicos e institucionales, relacionándose con el acceso a créditos, por lo que se pueden evidenciar vínculos entre las características de un producto innovador, como por ejemplo, el índice educativo es importante, porque el productor va a presentar características más amplias que le llevarán a tomar la decisión de adoptar una estrategia novedosa viable.

Las características aquí descritas delimitan la adopción, en esa perspectiva González y Sacco (2015) evidencian que las diferentes características pueden impactar el nivel de renta y por tal motivo calidad de vida de las familias, por otro lado, cuanto mayor el nivel de ingresos, mayor será la calidad de vida. Simioni y Zilliotto (2013) en su investigación realizada en el meso región del 
oeste de Santa Catarina relatan que al evaluar la influencia de las innovaciones tecnológicas en unidades de producción familiares, tomando como variables la renta, ser cooperado, el uso de redes sociales (internet), la escolaridad, la edad, el tamaño de la propiedad y el acceso a crédito, llegaron a la conclusión de que la adopción de las innovaciones está relacionada fuertemente con el capital y la tenencia de tierra, y los productores con mayor estudio conquista mayor ascenso (mejora en la propiedad, maquinarias modernas, etc.).

\subsection{Condiciones políticas}

Las políticas públicas influencian en las adopciones en cuestión, siendo así importante difundir con vistas a inhibir o suspender algunas, por lo que la agricultura familiar se ve influenciada por criterios organizacionales instruidos por policías públicas que no pueden ser controladas por los productores familiares como: educación, asistencia técnica, comportamientos de los precios en el mercado, acceso a crédito, impuestos, tasas, logística.

Es importante destacar que la asistencia técnica es una herramienta importante y promueve confianza junto al productor al adoptar una innovación. Este servicio puede ser prestado tanto por empresas públicas como privadas, pero casi siempre el productor familiar tiene acceso limitado a la asistencia técnica privada, además buena parte de técnicos en el campo no dominan bien la realidad de la agricultura familiar y con las alternativas adaptadas que se caracterizan para los productores de bajos ingresos.

El crédito para los productores familiares es un instrumento de política pública que tiene por objeto proporcionar recursos financieros al productor para cubrir los costos de operación y recorrer nuevas explotaciones agrícolas (innovaciones), y el distanciamiento del crédito por los productores puede perjudicar sus rendimientos de producción en la evolución de los mercados.

\subsection{Globalización}

Surgió a partir de la evolución uniforme de tres variables exógenas que según Wejnert (2002) son: tecnología global, conexiones mundiales e institucionalización. Donde la primera y segunda variable se articulan cuando las condiciones externas de los actores conducen sus comportamientos, es decir, tienen buena influencia en la toma de decisiones, cabe recordar que la globalización está ligada a los factores culturales de todos los países que tiene un papel importante en los efectos ocasionando mejorar o deteriorar el proceso de difusión de una adopción.

\subsection{Intermediación}

La intermediación cumple un papel importante en la adopción porque las principales entidades que son empresas de investigación privada (Universidades, ventas de maquinaria, insumos) o públicas (Universidades, EMBRAPA, CORPOICA, entre otras) no logran transferir completamente la innovación al productor familiar para tener una buena tasa de adopción. Según Howells (2006) el papel importante de un intermediario es ayudar a que la innovación se transfiera, y en el campo de la agricultura familiar es importante identificar al intermediario como un conjunto de conocimientos que van a ser utilizados por los productores para proporcionar soluciones cotidianas en el campo y, según el autor, los intermediarios pueden utilizar algunos métodos para mejorar el bienestar del productor, estos métodos se pueden observar en el cuadro 3:

\section{Cuadro 3. Fases de una adopción de una innovación.}

\begin{tabular}{|l|l|}
\hline Método & Concepto \\
\hline Previsión y Diagnóstico & Agrupación de funcionalidades para conocer más a fondo un problema que genera algún cambio. \\
\hline $\begin{array}{l}\text { Digitalización y procesamiento } \\
\text { de información }\end{array}$ & $\begin{array}{l}\text { Recoger informaciones sobre los principales cuellos de botella de los productores familiares, y enviarlos a } \\
\text { la empresa para crear o modificar la innovación. }\end{array}$ \\
\hline Combinación & $\begin{array}{l}\text { Combinar la función de la innovación con los productores familiares, y si ésta presenta algún problema, } \\
\text { recombinar con el productor para adquirir mejor uso de la innovación. }\end{array}$ \\
\hline
\end{tabular}




\begin{tabular}{|l|l|}
\hline Prueba de validación. & $\begin{array}{l}\text { Prueba o validación en las innovaciones que van a ser aplicadas con los productores rurales para mitigar los } \\
\text { fracasos de la adopción. De acuerdo con Howells (2006) es importante que la empresa adquiere credibilidad } \\
\text { por medio de validaciones de las innovaciones. }\end{array}$ \\
\hline Validación y regulación & $\begin{array}{l}\text { Vinculación das la empresa innovadora y la empresa u organización de intermediación. Para Howells (2006) } \\
\text { esta etapa es compleja porque se tiene que respetar los papeles de la innovación, por ejemplo, en el texto de La } \\
\text { compañía de planificación del Distrito Federal (2015), el Ministerio de Agricultura, Ganadería y Abastecimiento } \\
\text { Brasil (MAPA- Brasil) lanzó un programa del sello orgánico, lo cual fue una oportunidad de innovación para } \\
\text { que los productores pudieran obtener mejores precios y se posicionaran en el mercado. Esta política pública } \\
\text { se rige por la Ley 10831/2013, pero su aplicación se dio a partir del Decreto 6323, de 27 de diciembre de } \\
\text { 20o7, en el cual el principal objetivo es garantizar al productor un diferencial de mercado, y al comprador } \\
\text { calidad en los alimentos. Cuando los productores adoptan la innovación del sello se observa el aumento de } \\
\text { las ventas finales de sus productos orgánicos, pero aún se presentan cuellos de botella para el productor } \\
\text { como alto costo, transporte, falta de asistencia técnica, crédito, entre otros, ocasionando que el productor } \\
\text { adopte dicha innovación poco a poco. }\end{array}$ \\
\hline
\end{tabular}

Fuente: Adoptado de Howells (2006)

Adopción: En la agricultura familiar la adopción de una innovación puede ser el momento máximo de una aplicación de investigaciones, pasando a ser una práctica común en su actividad del día a día, de acuerdo con Rogers (1995), la adopción es la consecuencia de una metodología de decidir cómo se puede evidenciar en cinco procesos: comprensión, persuasión, decisión, ejecución y validación. Según Fujisaka (1994) existen cuatro razones relevantes para que los agricultores no adopten innovaciones destinadas a mejorar sus condiciones de vida las cuales son; cuando la innovación es parecida a la ya practicada por los productores familiares; si la innovación no se sincroniza con las condiciones de los productores sea geográfica, social o económica; cuando la difusión de la innovación no satisface la necesidad del productor y; cuando la innovación se destina a los productores sin las características y exige mucha inversión.

\section{Conclusiones}

La adopción para innovar en los agricultores familiares se muestra compleja, ya que esta presenta numerosas variables de aspecto político, social, económico e institucional y se deben tener en cuenta las variables en el momento de realizar una difusión. Hasta el momento la mayoría de las empresas innovadoras no consigue incorporar totalmente las interacciones sociales (características de un innovador), pues implica en un número mayor de productores y sus interacciones, en los cuales los comportamientos serán diferentes. Por eso es necesario que se desarrollen metodologías para trabajar con los productores familiares en las relaciones personales, para llegar con éxito a la adopción de una innovación, satisfaciendo e involucrando las necesidades de la familia

En la agricultura familiar es importante incorporar características que tengan como objetivo productividad, ganancia de escala y que la innovación se adecue a las segmentaciones de las características de los innovadores, de esa forma se puede alcanzar amortizar el esfuerzo para dotar una innovación. Por otro lado los productores no cuentan con mecanismos para protegerse de impactos económicos negativos, por lo tanto deben ser cautelosos para tener bajo riesgo de pérdidas en la adopción de técnicas novedosas.

sI el productor familiar participa de grupos como asociaciones, cooperativas, condominios y firmas en el proceso de la aceptación de una innovación, esta se puede obtener más fácil en referencia a los costos para innovar, porque generalmente las asociaciones están compuestas por productores con un mismo objetivo.

Es importante entender las nuevas luchas por la garantía de derechos, que involucran etnia, género y medio ambiente, donde estas son indispensable para las innovaciones agropecuarias en Latinoamérica, puesto 
que aunque estos temas pueden parecer indiferentes, influencian en las adopciones donde no se puede negar la explotación a la que están expuestas algunos grupos sociales, por ejemplo: las mujeres agrícolas pobres y de etnia negra. Se trata de una relación en la que clase y género se entrelazan en la profundización de las desigualdades, conllevando a la hegemonía de los intereses de los latifundiarios.

Asimismo, se concluye que los intermediarios tienen un papel fundamental en la asimilación de una innovación, ya que tienen un amplio conocimiento sobre el público, permitiéndoles realizar la transferencia de conocimientos de una manera adecuada y satisfactoria.

En este contexto, se puede afirmar que es de extrema importancia que las universidades y entidades de investigación realicen alianzas con instituciones intermediarias para difundir los conocimientos adquiridos. Es importante dejar claro las responsabilidades de cada socio, que cada empresa desarrolle su papel, para no tener fracaso en la adopción de la innovación. En lo que se refiere a las políticas públicas, es ciertamente imprescindible adaptar renovaciones en el ambiente organizacional e institucional para crear incentivos de créditos, subsidios, adquisición de tierras para mitigar la migración rural.

\section{Referencias bibliográficas}

Abramovay, R. (1998). Agricultura familiar e serviço público: novos desafios para a extensão rural. Cadernos de Ciência \& Tecnologia, Brasília, v.15, n.1, p.137-157.

Balsan, R. (2006). Impactos decorrentes da modernização da agricultura brasileira. Campo-Território: revista de geografia agrária, v. 1, n. 2, p. 123-15.

Cavalcanti, C. D., Riveros, J. L. T., \& Junior, N. N. G. (2020). Estado capitalista, sociabilidade capitalista: o impasse da luta por direitos humanos no Brasil. Humanidades \& Inovação, 7(17), 514-524.
Chayanov, A. (1975). Sobre la teoría de los sistemas económicos no capitalistas. Cuadernos Políticos, México D.F., n 5, p. 15-31.

Companhia de Panejamento do Distrito Federal. (2015). Diretoria de Estudos e Políticas Sociais. Mercado de produtos orgânicos: mecanismos de controle. Brasília

Empresa Brasileira de Pesquisa agropecuária. (2003). Pesquisa, desenvolvimento e inovação para o agronegócio brasileiro: cenários 2002-2012. Embrapa, Secretaria de Gestão e Estratégia, Brasília: Embrapa Informação Tecnológica, p.92.

Fujisaka, S. (1994). Learning from six reasons why farmers do not adopt innovation in tended to improve sustainability of upland agriculture. Agricultural Systems. Graet Britain, v. 46, n. 4, p. 409-425.

Gardner, A. L.; Oliveira, J. S. , (1984). Pesquisa aplicada: geração x adoção de tecnologia. Cadernos de Difusão de Tecnologia. Brasília, v.1, n. 2, p. 245263.

Goldennberg, J.; Libai, B.; Muller, E. 2001. Talk ofthe network: a complex systems look at the under lying process of word-of-mouth. Marketing Letters, v. 12, n. 3, p. 211-23.

Gonçalves, L. M., Godoy, C. M. T., de Oliveira Vargas, T., da Rocha Campos, J. R., \& Viganó, C. (2021). Como agricultores familiares compreendem a agroecologia? Um estudo de caso em VitorinoPR. Agricultura Familiar: Pesquisa, Formação e Desenvolvimento, 14(2), 29-49.

González, J.; Sacco, F. (2015). Estrategias de reproducción social de la producción familiar en la región fronteriza de Cerro Largo, Uruguay. Agrociencia Uruguay, v. 19, n. 2, p.101-109. 
Gopalakrishnan, S.; Damanpour, F. (1997) .A review of innovation research in economics, sociology and technology. Omega, v. 25, p. 15-28.

Hall, A.; Bockett, G.; Taylor, S.; Sivamohan M. V. K.; Clark, N. (2001). Why Research partner ships really matter: innovation theory, institutional arrangements and implications for developing new technologies for the poor, World Development, v. 29, n. 5, p. 783-797.

Howells, J. (2006). Intermediation and the role of intermediaries in innovation. ResearchPolicy, $\mathrm{n}$. 35, p. 715-728.

Lima S. M. V.; Castro, A. M. G. de; Mengo, O.; Medina, M.; MAESTREY, A.; Trujillo, V.; Alfaro, O. (2001). La dimensión de entorno en la construcción de la sostenibilidad institucional. Serie Innovación para la Sostenibilidad Institucional. San José, Costa Rica: ISNAR, 142p.

Lima, S. M. V.;Vieira, L. F.; Castro, A. M. G. de; Sarmento, E. P. de M. (2013). Juventude Rural e as Políticas e Programas de Acesso à Terra no Brasil: recomendações para políticas de desenvolvimento para o jovem rural. Brasília: MDA/NEAD, 182p.

Mahala. (2021). Tipos de inovações. https://agendamahala. com/tipos-de-inovacao.Acesso 13 abril 2021

Moraes, R. E., Soares, M. F., Noschang, J. P., Rodrigues, D. S., da Silva, D. S. C., Kommling, S., ... \& Silveira, I. D. B. (2020). Produção de carne ovina sob a ótica bem-estar animal. Brazilian Journal of Development, 6(4), 21900-21911.

Moraes, L. L., \& da Silva Nascimento, N. M. (2021). Mulheres rurais nordestinas e desviantes: um estudo sobre a quebra das expectativas de gênero no meio rural. Amazônica-Revista de Antropologia, 12(2), 725-747.

North, D. C. (1991) Institutions. The Journal of Economic Perspectives, v. 5, n. 1, p. 97-112.
Orduz-Rodrìguez, J.O.; Arango-Wisner, L.; Monroy, J. H.; Fischer, G. (2006). Comportamiento de la mandarina Arrayana en seis patrones en suelos ácidos del piedemonte Llanero de Colombia. En: Colombia. Agronomía Colombiana. Universidad Nacional De Colombia Facultad De Agronomía. p. $266-273$.

Organização das Nações Unidas para Alimentação e Agricultura. 2013. Disponibilidade de leite: produção e demanda atual, e perspectivas de médio prazo. In:___ Leite e produtos lácteos na nutrição humana. Brasil.

Ploeg, J. (2009). Sete teses sobre a agricultura camponesa. In: ___ Agricultura familiar camponesa na construção do futuro. Rio de Janeiro, p. 17-31.

Rodrigues, T. C., Leitão, F. O., Thomé, K. M., \& Cappellesso, G. (2021). Sharing economy practices in agrifood settlements: Integration of resources, interdependence and interdefinition. Journal of Cleaner Production, 294, 126357.

Rogers, E. M, (1995). Diffusion of Innovations. 3. ed.; New York: The Free Press, 453p.

Simioni, F. J.; Zilliotto,T. C. (2013). Influência das inovações tecnológicas em unidades de produção familiares da região oeste de Santa Catarina. Revista de economia e agronegócio, v. 10, n. 3, p. 419-440.

Souza Filho, H. M. de; Buainain, A. M.; Silveira, J. M. F. J. da; Vinholis, M. de M. B. (2011). Condicionantes da adoção de inovações tecnológicas na agricultura. Cadernos de Ciência \& Tecnologia, Brasília, v. 28, n. 1, p. 223-255.

Thomé, K. M., Cappellesso, G., Ramos, E. L. A., \& de Lima Duarte, S. C. (2020). Food supply chains and short food supply chains: coexistence conceptual framework. Journal of Cleaner Production, 123207. 
Triana Riveros, J. L. (2019). Campo ou cidade: influências sobre a escolha dos jovens rurais granadinos no estado do Meta, Colômbia. Tesis de maestría en agronegocios en la Universidad de Brasilia, 117. pag.

Valentino, V. (2007). Why are cooperatives important in agriculture? And organizational economics perpective. Journal of institutional Economics, v.3 n.01, p. 55-62.

Van Everdingen, Y. M.; WAARTS, E. (2003). The effect of national culture on the adoption of innovations. Marketing Letters, v. 14, n. 3, p. 217-232.

Wanderley, M., de N., B. (2003). Agricultura familiar e campesinato: rupturas e continuidade. Estudos sociedade e agricultura, n. 21, p.42-61.

Wejnert, B. (2002). Integratin gmodels of diffusion of innovations: A conceptual frame work. Annual Reviews, v. 28, p. 297-326. 